\title{
TTR
}

Traduction, terminologie, re?daction

\section{Dire les sciences et décrire les sens : Entre vulgarisation et lexicographie, le cas des dictionnaires de sciences}

\section{François Gaudin}

Volume 8, numéro 2, 2e semestre 1995

Technolectes et dictionnaires

URI : https://id.erudit.org/iderudit/037215ar

DOI : https://doi.org/10.7202/037215ar

Aller au sommaire du numéro

Éditeur(s)

Association canadienne de traductologie

ISSN

0835-8443 (imprimé)

1708-2188 (numérique)

Découvrir la revue

Citer cet article

Gaudin, F. (1995). Dire les sciences et décrire les sens : Entre vulgarisation et lexicographie, le cas des dictionnaires de sciences. TTR, 8(2), 11-27.

https://doi.org/10.7202/037215ar

\section{Résumé de l'article}

Dire les sciences et décrire les sens : Entre vulgarisation et lexicographie, le cas des dictionnaires de sciences - L'étude des stratégies rédactionnelles utilisées dans les dictionnaires spécialisés scientifiques permet de réfléchir aux façons de faciliter l'appropriation des connaissances. Ainsi, pour éviter les pièges tendus par des signifiants connus aux signifiés inédits, les relations lexicales sont précieuses, en particulier l'isonymie. Sur le plan syntagmatique, les relations predicatives sont utiles pour cerner la signification sur laquelle s'appuie la construction notionnelle. Les stratégies sont à contraster dans la mesure où les branches du savoir possèdent des traditions dénominatives diverses, dont une meilleure connaissance serait nécessaire tant pour une bonne description de ces vocabulaires que pour améliorer les propositions en matière d'aménagement terminologique.
Tous droits réservés @ C TTR: traduction, terminologie, rédaction — Les auteurs, 1995
Ce document est protégé par la loi sur le droit d'auteur. L'utilisation des services d'Érudit (y compris la reproduction) est assujettie à sa politique d'utilisation que vous pouvez consulter en ligne. 


\section{Dire les sciences et décrire les sens: Entre vulgarisation et lexicographie, le cas des dictionnaires de sciences}

\section{François Gaudin}

Nous nous intéresserons ici aux dictionnaires des sciences; $c$ 'est dire que nous nous situerons à la croisée des chemins entre diffusion du savoir et lexicographie spécialisée. La diffusion du savoir ne saurait être ici résumée à la seule problématique de la vulgarisation scientifique, car les auteurs de dictionnaires se démarquent de l'ensemble des pratiques rassemblées sous le terme de vulgarisation par une exigence d'exactitude dont témoignent, au plan éditologique (sur l'éditologie, cf. Baudet, 1991), les cautions scientifiques dont s'entourent les responsables d'ouvrages.

Nous parlerons ici de lexicographie spécialisée. Cette lexicographie spécialisée relève d'un genre hybride. Le primat qu'elle accorde à la dimension notionnelle la rapproche de la terminographie et de la conception de dictionnaires encyclopédiques. Ces derniers visant à rendre compte de façon exhaustive de l'ensemble des savoirs (Grand Dictionnaire Encyclopédique Larousse), nos dictionnaires de sciencesne peuvent prétendre à la même exhaustivité. En revanche, la terminographie travaille pour des publics restreints et exigeants (cf. Hermans et de Schaetzen, 1991). Elle accorde le primat à l'exactitude notionnelle et se préoccupe au premier chef des correspondances inter-langues, plus que de l'accessibilité du savoir.

Ce souci, qui permet d'exclure de notre étude les ouvrages trop sommaires, relevant plutôt des catégories des glossaires ou des lexiques (selon Boutin-Quesnel et al., 1985), conduit les auteurs de ces ouvrages 
à emprunter ponctuellement à l'histoire, histoire de la langue, mais aussi histoire des sciences.

Dans quelle mesure la visée didactique des ouvrages conduit-elle leurs auteurs à intégrer à leurs discours un discours métalinguistique? Quelles stratégies textuelles et sémantiques sont mises en cuvre pour faciliter la construction des concepts? Quelle place les auteurs accordent-ils à la langue générale? Comment favorisent-ils le passage des notions de sens commun à des concepts locaux? Voici quelques questions qui orienteront notre propos.

\section{Présentation des ouvrages}

La plupart des auteurs de dictionnaires scientifiques que nous avons étudiés (liste en bibliographie) destinent leurs ouvrages à un lectorat diversifié et affirment chercher à diffuser le plus largement possible le savoir qu'ils détiennent: ce qu'ils souhaitent, c'est «atteindre des publics très variés [et] travailler à diminuer l'écart entre ignorance et connaissance» (Le Lionnais, pp. vi-vii), «répondre à l'attente de tout honnête homme, cultivé mais non spécialisé» (Lévy, p. vii), ou être «le guide du lecteur curieux n'ayant pas nécessairement une formation scientifique» (Salem, 1990, p. 9). Cela est vrai même d'ouvrages très spécialisés, dont la conception se rapproche de celle d'ouvrages terminologiques, notamment par le nombre de termes complexes enregistrés et la multiplication des homonymes. Or un tel souci, clamé si nettement, ne peut être sans conséquence sur les nomenclatures, les partis-pris rédactionnels et la façon de gérer les liens entre la langue commune et les vocabulaires des différentes disciplines abordées.

En adoptant une telle stratégie descriptive, les auteurs privilégient les distinctions notionnelles au détriment des liens linguistiques. Comme nous l'avons déjà montré (cf. Gaudin, 1992), de ce point de vue, l'opposition entre dictionnaires et encyclopédies fonctionne moins bien, le dictionnaire scientifique généraliste s'avérant plus proche, dans son discours, de l'encyclopédie que du dictionnaire étroitement spécialisé.

Dans l'ensemble vaste et divers des ouvrages étudiés, l'opposition la plus fructueuse réside dans le caractère uni- ou pluri- 
disciplinaire, dans la mesure où ce sont les ouvrages regroupant un vocabulaire hétérogène qui s'avèrent les plus soucieux de lisibilité. Mais il ne faudrait pas entendre par lisibilité leur seule qualité rédactionnelle. Nous voulons par là insister sur le fait que les auteurs cherchent, par des procédés divers, à faciliter l'appropriation des concepts par leurs lecteurs.

Dans un travail précédent, nous avions ainsi établi la proximité de deux ouvrages généralistes, le Dictionnaire des sciences et l'Encyclopedia Universalis, que réunissaient une même volonté vulgarisatrice et des stratégies linguistiques comparables. Nous avions montré comment se traduisait en pratique le désir de leurs auteurs de mettre en culture les concepts scientifiques. Par souci d'en rester à un domaine proche de la lexicographie et afin de tester ce qui est possible dans le cadre d'un ouvrage de taille modeste (moins de 500 pages), nous prendrons l'essentiel de nos exemples dans le premier de ces deux ouvrages.

\section{Mots inconnus et sens obscurs}

Dans la mise à disposition d'un savoir, le premier problème posé tient à la matérialité de la langue, aux formes linguistiques. Et ce n'est pas le moins difficile. Il importe ici de bien distinguer les formes graphiques ou sonores, les signifiants, et les formes de sens, les signifiés.

Les formes de l'expression, les signifiants, posent un problème spécifique; ils sont repérables: une trop grande densité en mots rares arrête très vite le lecteur. Cet écueil-ci est assez simple à contourner: il suffit d'une vigilance soutenue à l'égard du jargon. Pour ce faire, la paraphrase est une aide précieuse: «L'organogenèse, ou formation des organes, se manifeste...» (Salem, 1990, p. 313).

Mais il n'y a pas que dans la rencontre de mots isolés que les signifiants peuvent faire obstacle. Le niveau syntaxique ne peut être ignoré. En effet, il importe également de ne pas rédiger d'une façon trop axiomatique, surtout dans les premières lignes, afin de ne pas décourager le lecteur. Examinons ces deux énoncés: « $\mathrm{Si} f$ est une fonction définie sur un intervalle I», (Salem, 1990, p. 353), «On dit 
qu'un nombre x est la limite d'une suite de nombres $\mathbf{U}_{\mathrm{n}}$ " (Salem, 1990, p. 252). Les mots, pris isolément, ne font pas obstacle; mais la tournure, par son tour très didactique, peut détourner le lecteur qui ne se sentirait pas l'âme d'un lycéen prolongé.

La question est plus délicate en ce qui a trait aux formes du contenu, les signifiés. La rareté, ou l'étrangeté, tient alors non pas aux signes vus, mais à la compréhension des signes lus. En effet, certains vocables peuvent être familiers quant à leur graphie, mais nouveaux quant à leur sens. Par exemple, sous la plume d'un physicien, moment devient pour le profane un mot traître: voilà une forme de l'expression usuelle, mais dont le sens utilisé par l'auteur diffère radicalement de la signification du mot usuel. Moment se comporte alors comme un faux-ami. On est alors dans le cas du bilinguisme dont parle Stella Baruk (1992, p. 16): il y a homonymie et le lecteur doit en être conscient; au rédacteur de l'y aider, le cas échéant. Mais cet exemple ne concerne qu'un nombre limité de termes et, si la métaphore du bilinguisme est séduisante, en matière de difficultés de sens, les cas de figure sont nombreux et pour contourner ces écueils-là, les cartes n'ont pas encore été tracées. Notons qu'en revanche, c'est peut-être par une disjonction oublieuse de la langue commune que le Dictionnaire de physique des éditions Masson et Eyrolles dissocie les deux emplois que connaît percolation en physique statistique et en chimie (Mathieu), alors qu'il s'agit manifestement de deux sous-sens rattachés à la même unité lexicale.

Il serait facile et plaisant de montrer les difficultés que rencontrent les auteurs et de détailler de quelle façon ils contreviennent à leurs pétitions de principe par hermétisme, ou par souci de précision. Nous ne décrirons pas de grands naufrages. Nous préférons ici nous intéresser au cabotage qui, de notions en notions, permet au lecteur d'éviter les récifs. C'est pourquoi nous limiterons notre propos aux ressources linguistiques mises en œuvre par les rédacteurs. En premier lieu, nous examinerons leur utilisation des relations lexicales.

\section{Les relations lexicales}

La notion de valeur négative, que l'on doit à Saussure, est familière aux linguistes. Pour sa part, le locuteur profane sait empiriquement qu'une 
bonne façon d'appréhender un mot nouveau est de le rattacher à un réseau d'unités déjà connues au sein duquel il va se différencier. Ceci illustre cela. Mais le problème est plus délicat quand on ne dispose guère de points d'appui pour construire, par contraste, une nouvelle signification et, à partir de là, une nouvelle notion: on oppose facilement masure à chaumière ou maison, et autobus à autocar ou transport en commun; mais à quoi opposer phospholipides, ou neurotransmetteur?

Les relations lexicales les plus usuelles sont l'hyperonymie, l'hyponymie et l'antonymie: l'hyperonyme, c'est l'englobant, le genre; l'hyponyme, c'est l'englobé, l'espèce; l'antonyme, c'est le contraire. À ces relations, inégalement exploitées dans les dictionnaires scientifiques, nous ajouterons la relation d'isonymie, moins familière.

L'hyperonymie sera traitée ici sans finesse et considérée comme la relation genre / espèce, que l'on trouve exploitée dans la définition canonique, dite aristotélicienne. On trouve dans les dictionnaires scientifiques des définitions de ce type: «L'oscilloscope est l'instrument de mesure...» (Salem, 1990, p. 315); «L'ozone est un gaz d'odeur piquante» (Salem, 1990, p. 318). L'hyponymie n'est guère utilisée que dans les descriptions de noms de classe: par exemple, particules élémentaires renvoie à électron, proton, neutron; frottement à frottement interne et frottement externe.

La relation d'antonymie est moins productive. Précieuse dans le dictionnaire général, elle concerne souvent les verbes et les adjectifs. Dans un dictionnaire constitué presque exclusivement de noms, elle est peu opératoire, les relations d'oppositions ne fonctionnant guère ni sur le mode de la gradation (chaud/tiède/froid), ni sur celui de la réciprocité (acheter/vendre), ni sur celui de la complémentarité (mort/vivant). Toutefois, comme dans la langue quotidienne, les oppositions les plus fréquentes sont celles qui, unissant des complémentaires, comprennent plusieurs termes: grades, couleurs, état civil (célibataire/concubin/ marié/divorcé/veuf), etc. Ainsi conçue, l'antonymie se rapproche beaucoup de l'utile relation dite «d'isonymie». 


\subsection{L'isonymie}

Quel que soit le respect que l'on éprouve pour Aristote, la définition qui porte son nom, faite d'un genre et de différences spécifiques, suffit rarement. Les raisons en sont nombreuses et ont été étudiées par les sémanticiens et les métalexicographes (Celex, 1990). Nous insisterons ici sur le fait qu'elle s'avère modérément opératoire au plan cognitif.

Pour cerner la signification d'une unité, c'est de contrastes dont le lecteur a besoin, et la définition canonique ne lui fournit qu'un mode de construction de catégories, celui d'une spécialisation avec le genre, le définissant. Cela convient pour définir hybride par rapport à être vivant. Mais si le mot que l'on consulte s'inscrit dans un vocabulaire moins familier, la même stratégie ne fonctionne pas: affirmer que «les œstrogènes sont des hormones stéroïdes, à 18 atomes de carbone, synthétisées à partir du cholestérol» (Salem, 1990, p. 303) suppose que l'on s'adresse à un lecteur assez connaisseur en chimie.

C'est ici que se situe l'intérêt de la relation d'isonymie: nous entendons par là «toute relation unissant deux unités mises en concurrence, le plus souvent de même niveau, sans que l'on puisse poser une hiérarchie valable selon tous les points de vue» (Assal et al., 1992). Cette relation s'utilise couramment: pour définir paracétamol, mieux vaudra l'opposer à aspirine que le définir comme «médicament qui...» Cela permet de contraster plusieurs significations très proches et non hiérarchiques. D'une part, l'approche sémantique est alors plus fine; d'autre part, cela permet de rendre compte de relations de proximité en l'absence de relations hiérarchiques (c'est pourquoi nous préférons le terme d'isonyme à celui, utilisé également, de co-hyponyme). Par exemple, lisant l'article consacré au terme adaptation, le lecteur apprend que, quand elle concerne un être vivant, «cette adaptation individuelle, ou accommodation, n'est pas héréditaire». Il construit ainsi une relation d'opposition adaptation / accommodation, opposition qu'il peut relier à des traits pertinents (espèce $v s$ individu; héréditaire $v s$ accidentelle). On procède de façon comparable dans l'énoncé suivant: «Le maser est l'analogue du laser pour les ondes radio» (Salem, 1990, p. 263). La description de cette relation d'isonymie n'est pas exclusive des autres relations; elle permet notamment de situer les parties d'un même ensemble. Ainsi à manteau, on lit «Partie intermédiaire du globe 
terrestre, située sous la croûte» (Salem, 1990, p. 262), ce qui permet d'opposer croûte à manteau, isonymes, comme parties de l'ensemble globe.

La relation d'isonymie permet de mettre en lumière l'avantage que tire le rédacteur d'une présentation soucieuse de décrire les notions de façon groupée. Prenons un exemple parlant, que nous avons déjà étudié (Gaudin, 1992): plusieurs articles consacrés à peptide permettent de situer ce terme par rapport aux notions d'acides aminés, d'oligopeptides, de polypeptides, de protéines et de molécule. Ainsi, l'on permet au lecteur de construire les relations sémantiques d'hyperonymie (c'est une sorte de molécule), de «ensemble de» (formée de l'enchaînement d'acides aminés) et d'hyponymie (comprenant les oligopeptides et les polypeptides). Protéine est situé comme sous-classe de polypeptides.

\subsection{Les relations sémantico-syntaxiques}

On peut également rencontrer dans les textes d'autres types de relations, tout aussi précieuses, relations que nous qualifierons de sémantiques et syntaxiques à la fois. Ce sont, par exemple, les relations dites «d'objet typique», "d'action typique», "d'agent typique» (cf. Lerat, 1987 et 1988), utilisées dans les descriptions sémantiques en terminologie, auxquelles nous ajouterons la relation dite «d'application typique». Présentons-les rapidement. Ces rubriques permettent de recenser les collocations typiques d'une unité lexicale. Prenons un exemple: filtre bactérien.

action typique: épuration

objet typique: effluents urbains

agent typique: micro-organismes

application typique: dépollution

Nos quatre rubriques nous ont permis de renseigner le lecteur sur l'utilité du filtre bactérien: il sert à l'épuration des effluents urbains, par l'action des micro-organismes, pour la dépollution. Ces rubriques, empruntées à l'analyse sémantique utilisée en terminologie, n'ont pas à être remplies par les rédacteurs de façon systématique; telle n'est pas 
leur tâche. Mais elles peuvent servir de guide pour analyser les stratégies de rédaction.

De fait, on s'aperçoit que les renseignements qu'elles permettent de consigner importent dans l'optique de la "vulgarisation dictionnairique». Et l'on voit ici l'intérêt que présente une telle analyse sémantique pour modéliser les connaissances; les frontières entre analyse de textes et modélisation du savoir sont bien incertaines.

Ainsi, les renseignements fournis sous l'entrée accélérateur de particules permettent d'indiquer que l'action typique est «la collision à haute vitesse», l'agent typique «une sonde», l'objet typique «les particules élémentaires». Et dans ce cas précis, l'application typique (rubrique que nous avons utilisée pour la confection d'un Dictionnaire des bioindustries) n'a pas à être distinguée de l'objet typique puisque, le rédacteur commence ainsi son article: «Pour étudier les particules élémentaires...» (Salem, 1990, p. 12). On comprend alors que le domaine d'utilisation du terme est limité au domaine d'étude de ces particules. Remarquons d'ailleurs que, dans leurs choix de vedettes, les ouvrages font peu de place aux applications des concepts qu'ils décrivent. Autre exemple, le photon est classé comme particule élémentaire (c'est son hyperonyme), son action typique étant de transmettre les interactions électromagnétiques.

Si les relations sémantico-syntaxiquessont utiles à décrire, nous ne posons nullement que leur description puisse suffire à la description notionnelle, qui suppose un discours (cf. Auroux, 1990), ou en réduire la nécessité. Nous pensons seulement que le passage des mots de tous les jours aux mots d'un savoir doit s'appuyer sur un effort pour «faire du sens». Techniquement, nous pensons que la description de la notion doit être accompagnée, ou précédée, d'une mise en lumière du sens, ou de l'absence de sens, des mots proposés à la lecture. Le vulgarisateur doit être un passeur avant de se promouvoir capitaine. Et le seul lieu de passage entre le non-sens du profane et la connivence sémantique de l'entre-soi des spécialistes, c'est le langage. Car il faut reconnaître «l'importance constitutive d'une langue de savoir dans l'édification de ce savoir», pour reprendre la formule de Stella Baruk (1992. p. 20). 
Dans cet effort pour faire du sens, les relations linguistiques, lexicales ou sémantico-syntaxiques, permettent - en quelque sorte - de cerner la valeur sémantique des mots, de circonscrire les signifiés des signes concernés. Il s'agit là d'une description linguistique proposée par les rédacteurs, de façon non systématique mais selon leur sensibilité et la confiance qu'ils ont dans la langue. Il est évident que pour décrire la notion et offrir matière à conceptualiser au lecteur, il faut dépasser ce stade préalable et décrire la notion dans ses divers aspects. Mais, à notre avis, en fournissant de façon claire et plus systématique ces renseignements simples, les rédacteurs mettraient en lumière les propriétés principales des objets et concepts à décrire et faciliteraient le travail d'approche du lecteur profane.

Cette stratégie permet égalementd'envisager la prise en compte des liens entre vocabulaire ordinaire et mots savants dont la co-existence doit être pacifique, et dont les différences ne doivent pas être élevées au rang d'obstacle systématique.

\section{La langue commune et les mots propres}

Il convient de distinguer les différents cas de figure. Tout d'abord, nous l'avons dit, les termes utilisés dans les interactions langagières entre gens du même métier sont fréquemment inconnus du profane. Ces signes-là se repèrent à leurs seuls signifiants. La difficulté d'accéder aux mots des sciences et techniques dépend des disciplines. En effet, alors que l'on a longtemps raisonné en termes de «vocabulaires scientifiques et techniques» (comme s'il s'agissait là d'un tout homogène !), les sciences, les techniques, les disciplines présentent de grandes différences dans les habitudes de dénomination qui les caractérisent.

\subsection{De la diversité linguistique des disciplines}

On manque sur cette question de travaux de synthèse qui permettraient d'y voir clair. Cependant, il est possible de contraster, par exemple, la tradition de recours au fonds gréco-latin qui caractérise la médecine au goût de la métaphore qui caractérise les choix dénominatifs des physiciens ou à la tendance massive qu'ont les généticiens de recourir aux calques. 
De même, le poids de l'histoire pèse différemment sur les différents savoirs. La victoire du clan des anti-phlogistiques a permis à la chimie une seconde naissance: Guyton de Morveau, Lavoisier et leurs amis ont gagné leur pari. Du coup, le langage de la chimie se présente comme une brillante illustration des idées des encyclopédistes sur les rapports entre science et langage, ce que démontre l'importance de Condillac dans la pensée de Lavoisier (cf. Bensaude-Vincent, 1993). Et si l'on trouve effectivement des couches diachroniques dans les dénominations chimiques (cf. Mounin, 1981), cette histoire est, au bout du compte, brève et peu accidentée.

Il résulte de ces différences que l'effet d'étrangeté des signes dépend des branches du savoir et des attaches qu'elles ont ou non maintenues avec le vocabulaire courant. Ainsi, pour en rester au seul niveau des entrées, des termes comme optimisation, optique non linéaire, pavage, radical libre, par exemple, ne produisent pas d'effet d'étrangeté. En revanche, le recours à des formants gréco-latins peu productifs crée des obstacles: phéromone, ptéridophyte (ou cryptogame, ce qui n'est guère plus transparent). Il en est de même des abréviations (rem), des symboles $\left(W^{+}\right)$et des éponymes (loi de Planck, espace de Hilbert). Il faudrait d'ailleurs contraster la fréquence d'utilisation de ces éponymes selon les disciplines: la chimie, la physique cultivent la mémoire des grands ancêtres de cette façon, qu'elles utilisent les noms d'éléments (einsteinium, fermium, mendélévium, nobélium), d'unités (curie, watt, volt, newton), de nombres (nombre d'Avogadro), de formules (équation de Bernouilli), ou d'appareils (bec Bunsen). Mais observe-t-on les mêmes phénomènes dans d'autres disciplines?

Toujours est-il que les éponymes constituent dans le vocabulaire d'une spécialité une enclave assez comparable à celle que forment, dans la langue générale, les noms de marque (Gaudin, 1993, pp. 104-105). On les trouve donc en bon rang dans les nomenclatures des ouvrages, ce qui s'explique par le fait que, les éponymes fonctionnant de façon analogue aux noms propres, l'exigence d'univocité est satisfaite.

Les effets d'obstacle qui se situent au niveau des signifiants sont donc divers, et dans certains cas, ne peuvent être évités. Cependant, il existe un autre ordre de difficultés qui réside dans l'écart entre le sens 
général d'un mot et l'acception particulière qu'il prend dans un corps de connaissances.

\subsection{Des concurrences historiques et culturelles}

Dans certains cas, la concurrence des significations tient à l'évolution historique. Or l'analyse des relations sémantiques et lexicales, telle que nous l'avons envisagée plus haut, est d'une utilité limitée à la synchronie. En effet, une part des difficultés que rencontre le lecteur profane face à des définitions scientifiques tient à la concurrence historique entre des notions anciennes, devenues culturelles, et des notions exactes liées à un savoir, les deux portant le même nom. La difficulté réside alors dans l'articulation des deux notions.

Pour ce faire, certains rédacteurs font le choix de situer historiquement la notion qu'ils ont à décrire. Le lecteur est alors invité à passer de l'une à l'autre et ici l'histoire des sciences s'avère précieuse pour faire comprendre, en la rendant sensible, la transition. Par exemple, la notice consacrée à air (masse $d^{\prime}$ ), commence ainsi: «Jusqu'au début du siècle, les météorologistes associaient les changements de temps aux hausses et baisses de baromètre. Vilhelm Bjerknes comprit, dans les années 1910...) (Salem, 1990, p. 22). En commençant de cette façon, on invite le lecteur à passer d'un modèle issu du sens commun à une approche plus rigoureuse. Examinons la définition d'algue: «Les algues sont des végétaux essentiellement aquatiques capables de photosynthèses». Elle procède d'une façon comparable en ce qu'elle permet de mettre à distance le stéréotype attaché au mot (les algues sont essentiellement aquatiques, et non plus exclusivement, comme c'est le cas pour le sens commun et le Petit Robert) et d'insister sur leur faculté de photosynthèse.

Dans le cas d'antibiotique, son caractère culturel indéniable le limite à la désignation de médicaments. L'article consacré à cette entrée la décrit comme «toute substance chimique produite par un micro-organisme, le plus souvent un champignon, ou par extension toute substance de synthèse, capable de ralentir la croissance des bactéries (bactériostatique) ou de les détruire (bactéricide)». En la décrivant ainsi, on permet au lecteur de prendre en compte le stéréotype qui est associé au mot tout en lui permettant de l'enrichir. 
On voit ici que l'importance de la dimension diachronique n'implique pas nécessairement une histoire inscrite dans le long terme. Elle concerne l'inscription du fonctionnement sémantique d'une unité lexicale dans une dualité de valeurs, l'une installée dans la sémiotique culturelle du vocabulaire commun, l'autre réduite à un sous-code partagé par une communauté socio-linguistique, et spécialement socioprofessionnelle, réduite.

\subsection{Pièges et vertus de la morphologie}

Enfin, les divergences sémantiques peuvent tenir à la morphologie elle-même. Les dénominations concernées sont en majorité des mots construits: des dérivés, composés, ou recomposés. Si ces formes sont motivées, leur signification étant analysable, leur formation peut induire en erreur. Revenons un instant sur antibiotique. L'article permet de cerner tout de suite l'objet typique des antibiotiques: les bactéries. Or ce que véhicule la morphologie, le sens prédictible du mot, renvoie à "contre la vie», par paraphrase des formants. Ce sont là des formants productifs et donc facilement insérables dans des paradigmes. À défaut de comprendre le mot, on le cerne en combinant les valeurs de anti et bio dans l'ensemble des mots dans lesquels ils apparaissent. Ce signifié élémentaire, un peu frustre, joue assurément un rôle dans le sens qu'acquiert cette forme linguistique en circulant dans le corps social. De la même façon, la dénomination anxiolytique, que nous avons étudiée, tend à induire en erreur dans la mesure où l'anxiolytique permet de combattre l'angoisse, et non l'anxiété, la distinction entre les deux reposant sur l'opposition somatique/non somatique (cf. Kernbaum). Et «c'est bien contre les manifestations somatiques de l'angoisse que luttent les anxiolytiques, même si des raisons morphologiques incitent à relier anxiolytique et anxiétén (Gaudin, 1992, p. 125). Ces pièges peuvent paraître anodins, mais ils gagneraient à être mieux étudiés par les linguistes et mieux perçus par les rédacteurs. Au stade de la rédaction d'un ouvrage de référence, on ne peut certes plus agir sur la qualité de l'équipement linguistique d'une discipline; mais si la sensibilité à ces questions se développait, c'est l'ensemble des habitudes linguistiques qui s'en verraient peu à peu transformées.

Cependant, la morphologie n'est pas faite que de leurres. Dans le cas d'agrégation, la définition, "mécanisme de formation des 
colloïdes, des gels et des suspensions à partir du collage de petites particules", permet de comprendre que le mot ici est un nom d'action (action d'agréger), le corps du texte permettant de relier le terme au nom de résultat: agrégat. C'est alors une famille morphologique qui est convoquée, ou plutôt, un paradigme dérivationnel.

L'article consacré à aromatique commence ainsi: «À l'origine, l'appellation aromatique, (du grec aroma, «parfum») était réservée à des composés naturels de la famille du benzène possédant un arôme caractéristique» (Salem, 1990, p. 47). En expliquant l'origine de la désignation, on permet d'intégrer, en le mettant à distance, le sens régulier de l'adjectif. En passant de l'adjectif au nom, le sens se spécialise, mais le trouble d'un autre sens, co-présent mais non évoqué, est évité.

De même, une dénomination comme bifurcation présente un écart par rapport à l'ensemble de ses significations usuelles. Cependant, la notion de "changements qualitatifs dans l'évolution» s'intègre aisément dans l'évolution historique du mot qui lui a conféré sa capacité à dénommer des réalités abstraites: on passe de l'embranchement à l'alternative. Il y a donc ici continuité, le passage devant être souligné afin d'introduire la spécificité du sens mathématique sur le terreau du sens général. Ce mouvement de spécialisation de dénominations usuelles est parfois perçu comme une gêne. Or il peut au contraire constituer une aide, la signification permettant une sorte de «pré-découpage cognitif».

Prenons l'exemple de percolation, que nous avons déjà évoqué rapidement ailleurs (Gaudin, 1992, p. 126). Le mot ne figure pas à la nomenclature du Petit Robert (1986), pas plus que le verbe percoler. C'est donc un terme technique. Cependant, il figure dans la même famille que percolateur, mot courant. Une explication prenant en compte la morphologie rassemble les trois mots dans une même famille: on a là un verbe, percoler, un nom d'instrument, percolateur, et un nom d'action, percolation. Et, de fait, le percolateur présente bien «une situation de type percolation» (Salem, 1990, p. 322). Les ressources de la langue fournissent donc ici la possibilité de construire la notion scientifique à partir d'un objet, et d'un nom, de la vie quotidienne. 


\section{Conclusion}

Un tel crédit accordé à la langue commune est assez rare dans le discours scientifique: il y va de ce que Hilary Putnam appelle joliment la "charité dans l'interprétation». (1984, p. 135). Il s'agit en quelque sorte de réserver une place au découpage proprement linguistique, auquel il est fait crédit, en-deçà de la construction conceptuelle. De ce point de vue, la diversité des stratégies employées par les auteurs indique combien l'attitude vis-à-vis de la langue générale, souvent conçue comme trompeuse, constitue une ligne de fracture. Ainsi, parmi notre corpus, le Dictionnaire de biologie de Lender et alii se présente comme très proche d'ouvrages terminologiques; il ne connaît que des termes classés strictement en domaines, ne présente aucune marque métalinguistique et ne dessine jamais les rapports existant entre les noms des concepts qu'il étudie et la langue générale.

Il ne s'agit pas de stigmatiser un ouvrage: la défiance à l'endroit du langage est fréquente. Elle a motivé maints projets de métalangues depuis, au moins, Leibniz et elle résulte d'une confusion entre la signification des unités et leurs actualisations dans des opérations de désignation particulières. Or la langue n'est pas faite pour dire le vrai; la parole parfois y parvient. C'est à nos discours de construire le sens d'énoncés provisoirement acceptés. On peut user de termes cryptiques; cela peut avoir son utilité, cognitive, mais aussi sociale...

Toutefois, il faut savoir et faire savoir qu'une polysémie bien réglée facilite la construction des concepts au sein de discours. Et c'est à l'étude de ces discours qu'il convient de s'attacher. L'approfondissement des travaux en matière d'analyse linguistique, sémantique et morphologique, des vocables employés permettrait, en rendant sensible le rapport d'inclusion existant entre un lexique naturel et ses sous-ensembles, de mieux insérer les sciences et les techniques dans la langue générale, et par là de faciliter leur mise en culture. Ce peut être une des voies par lesquelles s'illustrerait une terminologie sociale qui ne serait coupée, ni des sciences du langage, ni de l'épistémologie, ni des besoins sociaux. 


\section{Références}

ASSAL, Allal et al. (1992). "Sémantique et terminologie: sens et contextes», Terminologie et traduction, 2/3, pp. 411-421.

AUROUX, Sylvain (1990). «Avant-propos». Encyclopédie philosophique universelle. II - Les notions. Paris, PUF, pp.

vii-xx.

BARUK, Stella (1992). Dictionnaire de mathématiques. Paris, Le Seuil, $1324 \mathrm{p}$.

BAUDET, Jean-Claude (1991). «Éditologie et sociolinguistique», Cahiers de linguistique sociale, 18, Rouen, éd. URA CNRS 1164, pp. 81-99.

BENSAUDE-VINCENT, Bernadette (1993). Lavoisier. Mémoires d'une révolution. Paris, Flammarion, 469 p.

BOUTIN-QUESNEL, Rachel et al. (1985). Vocabulaire systématique de la terminologie. Québec. Office de langue française, $38 \mathrm{p}$.

CELEX (1990). La définition. Paris, Larousse, 304 p.

Encyclopedia Universalis (1989). Paris.

GAUDIN, François (1992). «Terminologie et démocratisation du savoir: à propos de dictionnaires scientifiques», Le langage et l'homme, XXVII(2-3), pp. 123-129.

(1993). Pour une socioterminologie: des problèmes sémantiques aux pratiques institutionnelles. Rouen, Publications de l'Université de Rouen, $254 \mathrm{p}$.

HERMANS, Ad et Caroline de Schaetzen(1991). «Étude fonctionnelle des dictionnaires techniques par les méthodes de l'analyse de la valeur", Le langage et l'homme, XXVI(4), pp. 301-303. 
KERNBAUM, Serge, dir. (1982). Dictionnaire de médecine. Paris, Flammarion, $1024 \mathrm{p}$.

LE LIONNAIS, François, dir. (1983). Dictionnaire de mathématiques. Paris, P.U.F., 848 p.

LENDER, Théodore et al. (1979). Dictionnaire de biologie. Paris, P.U.F., 437 p.

LERAT, Pierre (1987). «Le traitement des emprunts en terminographie et néographie», Cahiers de lexicologie, 50, Paris, Didier-Érudition, pp. 137-144.

(1988). "Terminologie et sémantique descriptive», $L a$ banque des mots. Paris, CILF, pp. 11-34.

LÉVY, Pierre (1988). Dictionnaire de physique. Paris, P.U.F., 890 p.

MATHIEU, et al. (1985). Dictionnaire de physique. Paris, Masson \& Eyrolles, $567 \mathrm{p}$.

MOUNIN, Georges (1981). «Une sémiologie du système des signes de la chimie», Diogène, 114, Paris, Gallimard, pp. 92-104.

Petit Robert 1 (1986). Paris, Le Robert.

PUTNAM, Hilary (1984). Raison, histoire et vérité. Paris, Minuit, 242 p.

SALEM, Lionel (1990). Dictionnaire des sciences. Paris, Hachette, 496 p.

François Gaudin: Université de Rouen, IRED, 76821 Mont SaintAignan Cédex, France.

RÉSUMÉ: Dire les sciences et décrire les sens: Entre vulgarisation et lexicographie, le cas des dictionnaires de sciences - L'étude des stratégies rédactionnelles utilisées dans les dictionnaires spécialisés 
scientifiques permet de réfléchir aux façons de faciliter l'appropriation des connaissances. Ainsi, pour éviter les pièges tendus par des signifiants connus aux signifiés inédits, les relations lexicales sont précieuses, en particulier l'isonymie. Sur le plan syntagmatique, les relations prédicatives sont utiles pour cerner la signification sur laquelle s'appuie la construction notionnelle. Les stratégies sont à contraster dans la mesure où les branches du savoir possèdent des traditions dénominatives diverses, dont une meilleure connaissance serait nécessaire tant pour une bonne description de ces vocabulaires que pour améliorer les propositions en matière d'aménagement terminologique.

ABSTRACT: Writing Sciences/Describing Meanings: Between Vulgarisation and Lexicography, the Case of Science Dictionaries The study of the writing strategies used in specialized science dictionaries can further reflection on ways to facilitate the appropriation of knowledge. Lexical relations, in particular isonymy, are invaluable in resolving the difficulties which arise when common signifiers acquire new meanings. On the syntagmatic level, predicate relations are useful in identifying the meaning underlying the construction of notions. Strategies vary, since different branches of science have different denominative traditions. A better understanding of these traditions is necessary, not only to describe these specialized vocabularies better, but also to improve suggestions for terminological categorization. 